\title{
thell \\ Dedicated Software Enhancing Data-independent Acquisition Methods in Mass Spectrometry
}

\section{Aivett Bilbao ${ }^{\text {ab }}$, Frédérique Lisacek ${ }^{* b c}$,} and Gérard Hopfgartner ${ }^{\star a}$

${ }^{*}$ Correspondence: Prof. Dr. G. Hopfgartnera , E-mail: Gerard.Hopfgartner@unige. ch; Dr. F. Lisacek ${ }^{b}$, E-mail: Frederique.Lisacek@isb-sib.ch; aLife Sciences Mass Spectrometry, Department of Inorganic and Analytical Chemistry, University of Geneva, 30, quai Ernest-Ansermet, $\mathrm{CH}-1211$ Geneva 4; 'PProteome Informatics Group, SIB Swiss Institute of Bioinformatics, CMU, rue Michel Servet, CH-1211 Geneva; 'Computer Science Department, University of Geneva, Geneva

Keywords: Data-independent acquisition · Interference removal - Metabolomics · Proteomics · SWATH · Variable-precursorisolation-window widths

Mass spectrometry combined with liquid chromatography has become the main analytical platform to characterize proteins and low-molecular-weight compounds in complex samples. In proteomics and metabolomics, samples are commonly analyzed by data-dependent acquisition (DDA) methods. However, the intensity-based semi-stochastic selection of precursor ions favors highly abundant analytes while under-sampling the lowabundance ones. In contrast to this sequential detection, selection, and analysis of individual ions, alternative data-independent acquisition (DIA) methods systematically parallelize the fragmentation of all detectable ions within a wide mass range regardless of their intensity. The mass spectra recorded by DIA are a continuous map of multiplexed fragment chromatographic profiles of all detectable analytes. Therefore, DIA integrates qualitative and quantitative information, providing broader dynamic range of detected signals, improved reproducibility for identification, and better sensitivity and accuracy for quantification.

Despite these advantages, the concurrent fragmentation of analytes has the drawback of increasing the likelihood of

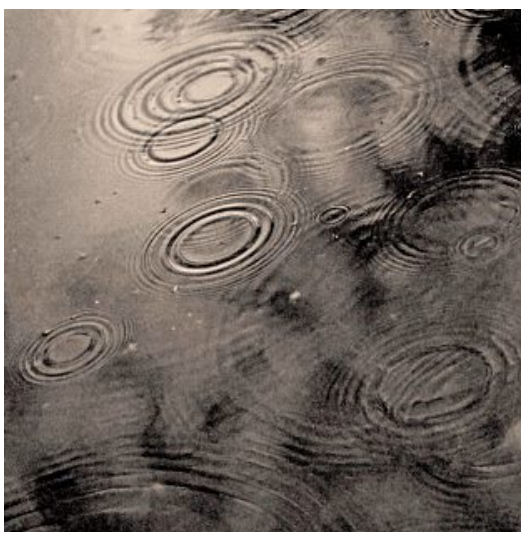

Analogy for cross fragment ion interference. Several waves causing interference to each other. Photo: quapan (https://www.flickr.com/photos/ hinkelstone/7001475448), sepia recolor. interference due to the overlap of fragment ions from different precursors. We developed two solutions to tackle this issue and further expand the benefits of DIA.

The first solution comprises a program called SwathTUNER, implemented to design and optimize different DIA methods using stepwise isolation windows (e.g. SWATH) The benefits of utilizing acquisition methods with variable-precursor-

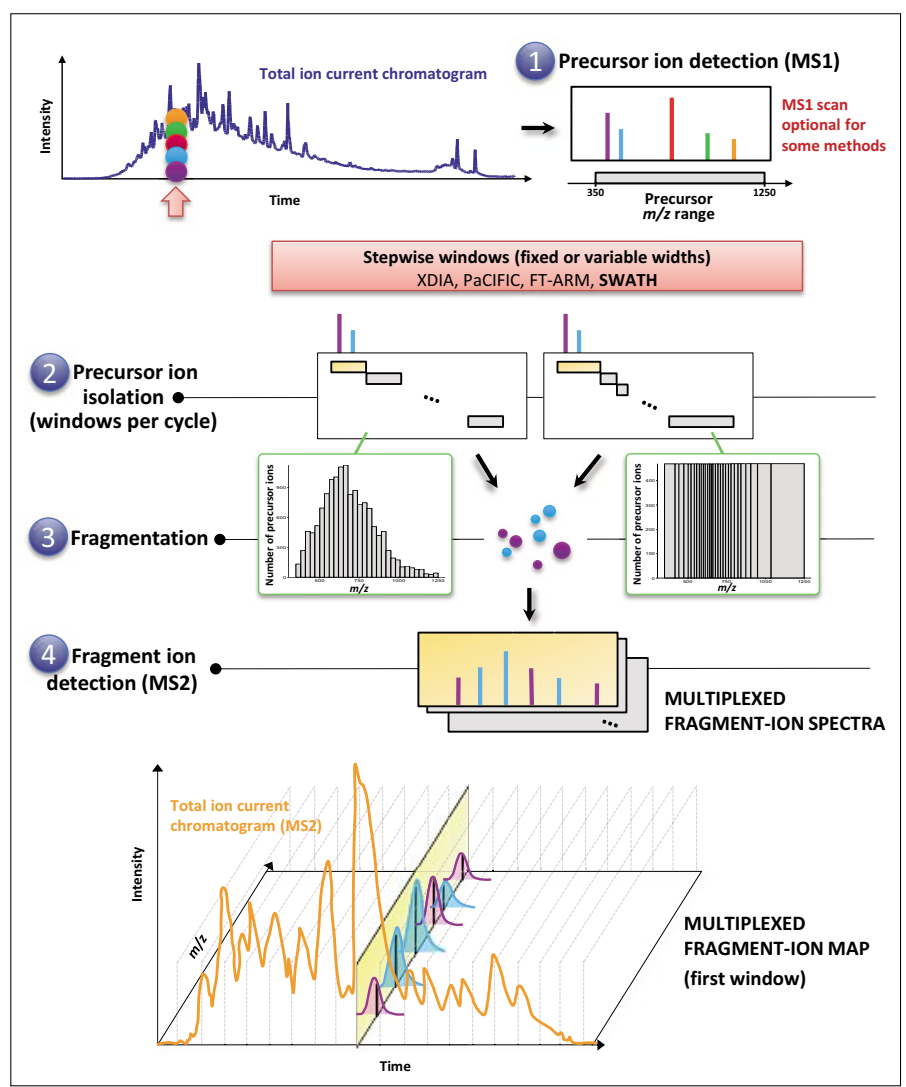

Data-independent acquisition using stepwise isolation windows. Green boxes show examples of the frequency distribution of all detected precursor ions in a run for methods using fixed or variable widths optimized with SwathTUNER.

isolation-window widths were demonstrated for the profiling of proteomic and metabolic samples.

The second solution is the 'non-outlier fragment ion' (NOFI) ranking algorithm. NOFI assigns low priority to fragment ions affected by interference during DIA acquisition. The optimal subset of high-priority fragment ions defined by NOFI effectively excludes interfered fragment ions from quantification. Improvement for label-free quantification was illustrated on a well-defined quantitative dataset and on a biologically relevant cell digest.

DIA methods coupled with adequate software solutions deliver comprehensive information in a single-shot analysis to study the full range of small to large molecules and unravel biological processes.

\section{References}

A. Bilbao, E. Varesio, J. Luban, C. Strambio-De-Castillia, G. Hopfgartner, M. Müller, F. Lisacek, Proteomics 2015, 15, 964

Y. Zhang, A. Bilbao, T. Bruderer, J. Luban, C. Strambio-De-Castillia, F. Lisacek, G. Hopfgartner, E. Varesio, J. Proteome Res. 2015, 14, 4359.

A. Bilbao, Y. Zhang, E. Varesio, J. Luban, C. Strambio-De-Castillia, F. Lisacek, G. Hopfgartner, J. Proteome Res. 2015, 14, 4581 . 